\title{
Experimental research on the continuous roll forming of three- dimensional surface parts
}

\author{
Qing-Min Chen ${ }^{1, a}$, Wei-Xue Hu ${ }^{1, \star}$ Xue-Ping Yan $^{1}$ \\ ${ }^{1}$ Roll Forging Research Institute, Jilin University (Nanling Campus), 5988 Renmin Street, \\ Changchun, P.R.China \\ *Corresponding author: huwxjlu@163.com \\ achenqm@jlu.edu.cn
}

Keywords: Continuous forming; Flexible roll; Rolling speed; Three-dimensional surface

\begin{abstract}
Continuous roll forming is a new sheet metal forming process for three-dimensional surface parts. In the process, the sheet metal is thinned non-uniformly in transverse direction by controlling the distribution of the gap between the upper and lower forming rolls, the longitudinal elongation is different, which makes the sheet metal generate a doubly curved surface parts. The rolling speed and the thickness of sheet metal etc. have great influence on the forming results. In this paper, a series of experiments of continuous roll forming have been carried out, the radii of curvature in the longitudinal and lateral directions were measured, and the effects of the rolling speed and the thickness of sheet metal are obtained.
\end{abstract}

\section{Introduction}

Continuous forming is a new kind of forming process for sheet metals, which can produce three-dimensional surface parts rapidly and efficiently, make a large reduce in the setup cost. At present, three-dimensional surface parts are widely used in the manufacturing of automobiles, ships, high speed trains and airplanes. The demand of market for product variety and product individuation has been growing rapidly, which prompts researchers to develop new forming process in order to realize rapid and lower-cost forming.

Continuous manufacturing technology is suitable for producing small-lot or single production as for the low set-up cost. Shim et al.[1] developed the line array roll set process which was composed of three rows of upper rolls and three rows of lower rolls, and each row of rolls was composed of multiple independent short rolls. The sheet metal was deformed by the line array roll set. Continuous flexible forming process for the fabrication of three-dimensional sheet metal parts was proposed based on the idea of roll-bending using flexible rolls [2,3]. The continuous flexible forming process employs three flexible rolls controlled at multi-point as forming tools, the rotations of the flexible rolls drive the sheet metal feeding and bend the sheet metal in the longitudinal direction and transversal direction simultaneously, and thus a three-dimensional surface is formed continuously.

To realize rapidly forming of three-dimensional surface part, rolling technique which is usually used in bulk forming is applied to sheet metal forming and a new process to manufacture curved surface parts, so-called continuous roll forming has been proposed [4-6]. The process combines rolling technique with the bendable rolls for manufacturing three-dimensional surface part [4], by controlling the roll gap between the upper and lower forming rolls, doubly bending deformation is generated. Cai et al. $[4,5]$ theoretically analyzed bending deformation of sheet metal in continuous roll forming process, the bending equations of longitudinal and lateral were set forth, and process design methods were given. In this paper, a series of experiments have been carried out, and the effects of the roll speed and thickness of the sheet metal are experimental studied.

\section{Principle of continuous roll forming}

As shown in Figure 1a, the system of continuous roll forming is composed of a pair of rolls, different three-dimensional surface parts can be formed by changing the curved contour of forming roll and distribution of roll gap. The gap between the upper and lower rolls is non-uniformly distributed in the transversal direction, the sheet metal is compressed non-uniformly so that non 
-uniform elongation in the longitudinal direction takes place and longitudinal strain non-uniformly distributed across transversal section is generated. The sheet mental bent in the transversal direction and non-uniform elongation in the longitudinal direction, a three-dimensional surface part is formed finally. With the rotations of the upper and lower forming rolls, the sheet mental is continuously shaped into a doubly curved surface. Each roll can be adjusted and bent with small deflection independently by a series of control points on it. Rolling equipment shown in Figure 1b.

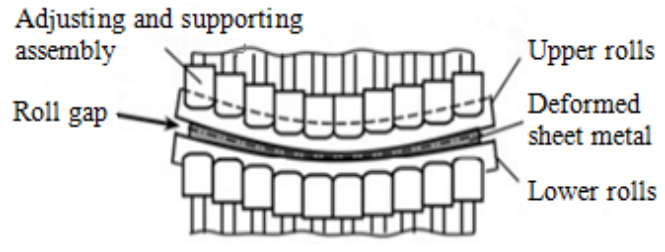

a. principle of continuous roll forming

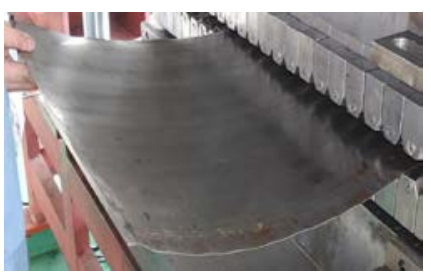

b. rolling equipment

Fig.1 Principle of continuous roll forming and rolling equipment

If the roll gap is relatively small in the middle and large on the two edges, a surface with positive Gaussian curvature will be obtained. If the roll gap is relatively large in the middle and small on the two edges, a surface with negative Gaussian curvature will be obtained.

\section{Influence of rolling speed}

In order to investigate the influence of the rotational speed of working roll on the curvature radius, experiments on the convex curved parts were carried out. The size of sheet is $400 \mathrm{~mm} \times 330 \mathrm{~mm}$ $\times 2.5 \mathrm{~mm}$, the material is pure aluminum. The bending radius of the lower roll is $40 \mathrm{~m}$, the maximum thickness reduction is $0.04 \mathrm{~mm}$, and the rolling parts formed with four speeds are shown in Figure 2.

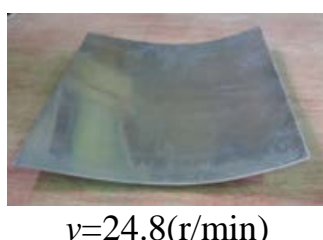

$v=24.8(\mathrm{r} / \mathrm{min})$

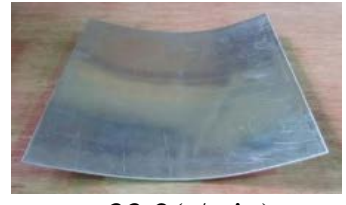

$v=33.2(\mathrm{r} / \mathrm{min})$

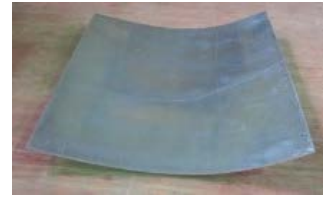

$v=41.5(\mathrm{r} / \mathrm{min})$

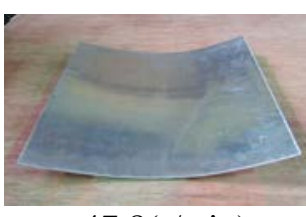

$v=47.8(\mathrm{r} / \mathrm{min})$

Fig.2 Pictures of flexible rolling parts at different rolling rotational speeds

In order to analysis the change of radius of the tansversal and longitudinal curvature, the measured results of flexible rolling parts at different rolling speeds were obtained and are shown in Table1. It can be seen that with the increase of rotational speed, the radius of longitudinal curvature decrease, the change of the radius of transversal curvature is not obvious. The the radius of curvature with rotational speeds is shown in Figure 3.

Table1 Measured results of flexible rolling parts at different rolling speeds

\begin{tabular}{ccc}
\hline Rotational speed $(\mathrm{r} / \mathrm{min})$ & Radius of transversal curvature $(\mathrm{mm})$ & Radius of longitudinal curvature $(\mathrm{mm})$ \\
\hline 24.8 & 1923.4 & 400.9 \\
\hline 33.2 & 2315.0 & 332.6 \\
\hline 41.5 & 2604.4 & 301.1 \\
\hline 47.8 & 2806.2 & 284.3 \\
\hline
\end{tabular}

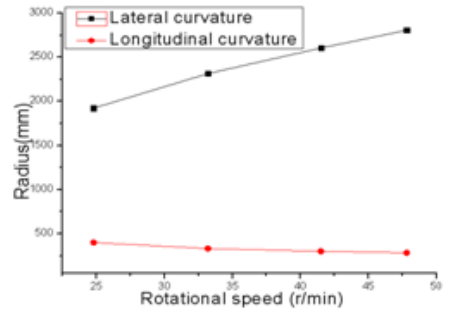

Fig 3 Variation of radius with rotational speeds

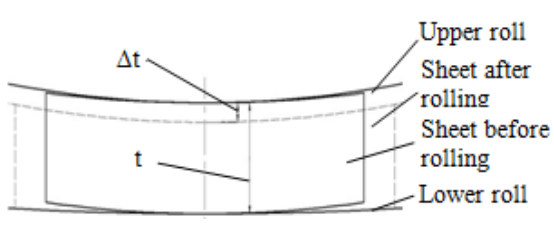

Fig 4 Schematic diagram of thickness change 
According to the experimental results, from $v=24.8(\mathrm{r} / \mathrm{min})$ to $v=33.2(\mathrm{r} / \mathrm{min})$, the rotational speed of rolling has obvious influence on the longitudinal curvature radius of forming parts. The larger the forming speed, the smaller the radius of the longitudinal curvature. The radius of the transversal curvature of the forming parts increase with the increase of the working rotational speed, but the increase proportion is smaller. The experimental results show that large rotational speed leads to large ratios of the transversal curvature radius to longitudinal curvature radius, at the same time, the curvature of the forming parts is small, the 3D surface close to the cylinder. Therefore, large rotational speed has a bad effect on rolling process. In the experimental process, as the rotational speed increase, it is more difficult for the operator to control the sheet metal. Therefore, if the forming efficiency can be ensured, a small rotational speed should be selected.

\section{Influence of sheet thickness}

In order to analysis the influence of sheet thickness on rolling, sheet metal in three thicknesses were selected. Figure 4 is the schematic digram of thickness before and after rolling. In the beginning, the lowest point of upper and lower rollers contact with the surface of the sheet, and the state of the sheet represent by the solid line, and the minimum vaule of roll gap equal to the thickness of the primary sheet, which represent by $\mathrm{t}$ in Figure 4. In the end of rollers movement in vertical direction, sheet thinning process completed, the profile represent by dasn line, the maximum amount of reduction is $\Delta t$.

Firstly, in the case of $\Delta t=0.04 \mathrm{~mm}$, pure aluminum sheets in three thickness were selected. In this experiment, convex curved surface were taken as an example. The upper and lower working roll is controlled by the lifting device. The rolling parts are shown in Figure 5. With the thickness variation, it can be found that the larger the thickness of the sheet, the larger the radius of the longitudinal curvature, the radius of transversal curvature increase as well.

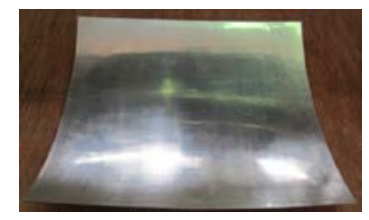

$t=1.5 \mathrm{~mm}$

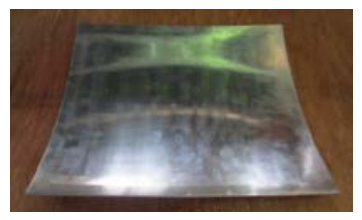

$t=2.0 \mathrm{~mm}$

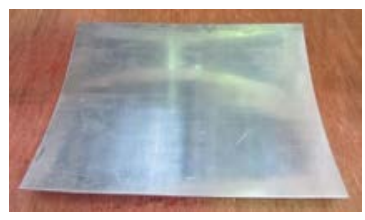

$t=2.5 \mathrm{~mm}$

Fig. 5 Forming parts of different thickness with $\Delta t=0.04 \mathrm{~mm}$

The radii of transversal and longitudinal curvature were obtained by a measuring method. The results are shown in table 2. According to the experimental results, with the increasing of the thickness of the sheet, the radii of transversal and longitudinal curvature of the rolling parts are increased too.

Table. 2 Measure results of different thicknesses forming parts with $\Delta t=0.04 \mathrm{~mm}$

\begin{tabular}{ccc}
\hline Sheet thickness $(\mathrm{mm})$ & Radius of transversal curvature $(\mathrm{mm})$ & Radius of longitudinal curvature $(\mathrm{mm})$ \\
\hline 1.5 & 1736.1 & 327.3 \\
\hline 2.0 & 1923.6 & 451.9 \\
\hline 2.5 & 2404.3 & 632.0 \\
\hline
\end{tabular}

The experimental results show that the radius of the transversal and longitudinal curvature of the forming parts increase with the increase of the thickness of the sheet. It appears that thick sheet resist to rolling deformation. In the rolling process, this phenomenon should be considered.

In the following experiments, the amount of maximum reduction is $\Delta t / t=1.8 \%$. According to these experiments, the thickness of the sheet metal increase, the bending radius of the rolling parts decrease and the amount of sheet metal thinning increase. As the lower work roll profile unchanged, the radius of the actual roll seam line of the curvature decrease. Sheet in three thicknesses are respectively $1.5 \mathrm{~mm}, 2.0 \mathrm{~mm}$ and $2.5 \mathrm{~mm}$, corresponding to the parts shown in Figure 6. From the forming parts pictures, the part in the thickness of $t=1.5 \mathrm{~mm}$ has a relatively small radius of longitudinal curvature. 
The radii of longitudinal curvature of the part $t=2 \mathrm{~mm}$ and $t=2.5 \mathrm{~mm}$ are increase in turn. Radii of transversal curvature changed in the same way.

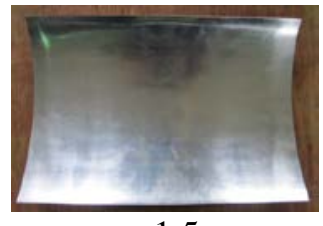

$t=1.5 \mathrm{~mm}$

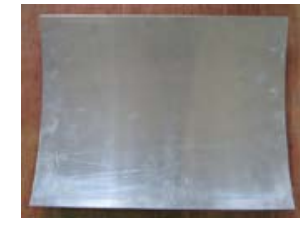

$t=2.0 \mathrm{~mm}$

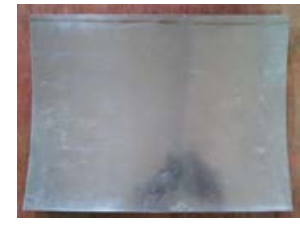

$t=2.5 \mathrm{~mm}$

Fig.6 The forming parts of different thicknesses with $\Delta t / t=1.8 \%$

Table.3 Measure results of different thicknesses forming parts with $\Delta t / t=1.8 \%$

\begin{tabular}{ccc}
\hline Sheet thickness $(\mathrm{mm})$ & Radius of transversal curvature $(\mathrm{mm})$ & Radius of longitudinal curvature (mm) \\
\hline 1.5 & 1902.9 & 452.8 \\
\hline 2.0 & 2170.4 & 468.1 \\
\hline 2.5 & 2460.9 & 495.9
\end{tabular}

According to the experimental results, the transversal and longitudinal curvature radius of the forming parts increase with the increase of the thickness of the sheet, which is similar to the experimental results with a constant $\Delta t=0.04 \mathrm{~mm}$. In the rolling process, plastic deformation produced in sheet mental. The deformation of thick sheet metal is insufficient with respect to thin sheet metal and the deformation resistance of the sheet mental becomes larger as well as the sheet thickness increase. So that with $\Delta t / t=1.8 \%$, the curvature radius change in a relative low degree. Analysis of the change of roll gap in these experiments, the radius of the middle curvature of the roll gap decrease with the increase of the sheet thickness, but the experiment results proved that the reduction of the radius of the roll seam does not affect the radius of the transversal curvature of the forming part. This proved that the thickness of sheet is the main factor that affects the curvature radius of the forming parts.

\section{Conclusions}

From the expriments, the conclusions are obtained as follow:

1. The larger the rotational speed, the smaller the radius of longitudinal curvature. The radius of transversal curvature of the forming parts increase with the increase of the rotational speed, but the increase proportion is smaller.

2. When the sheet thickness increase, the transversal curvature radius and longitudinal curvature radius increase.

\section{References}

[1] S. J. Yoon, D.Y.Yang. Development of a Highly Flexible Incremental Roll Forming Process for the Manufacture of a Doubly Curved Sheet Metal. CIRP Annals - Manufacturing Technology, Vol.52 (2003), p.201

[2] Z.Y. Cai, M.Z. Li and Y. W. Lan. Three-dimensional sheet metal continuous forming process based on flexible roll bending: principle and experiments. Journal of Materials Processing Technology, Vol. 212 (2012), p.120

[3] Z. Sui, Z.Y. Cai, Y.W. Lan. Simulation and software design of continuous flexible roll bending process for three dimensional surface parts, Materials \& Design, Vol.54 (2014), p.498

[4] Z.Y. Cai, M.Z. Li. Principle and theoretical analysis of continuous roll forming for threedimensional surface parts. Science China Technological Sciences, Vol.56 (2013), p.351

[5] Z.Y. Cai, M. Wang, M.Z. Li. Study on the continuous roll forming process of swept surface sheet metal part. Journal of Materials Processing Technology, Vol.214(2014), p.1820

[6] Z.Y. Cai, D.B. Guan, M. Wang. A novel continuous roll forming process of sheet metal based on bended rolls, International Journal of Advanced Manufacturing Technology, Vol.73(2014), p. 1807 